\title{
Dialogue Design for a Robot-Based Face-Mirroring Game to Engage Autistic Children with Emotional Expressions
}

\author{
P. Chevalier ${ }^{1}$, J.J. Li ${ }^{1}$, E. Ainger ${ }^{2}$, A.M. Alcorn ${ }^{2}$, S. Babovic ${ }^{3}$, V. Charisi ${ }^{1}$, S. Pe- \\ trovic $^{3}$, B.R. Schadenberg ${ }^{1}$, E. Pellicano ${ }^{2}$, V. Evers ${ }^{1}$ \\ ${ }^{1}$ Human Media Interaction, University of Twente, The Netherlands \\ ${ }^{2}$ Centre for Research in Autism and Education, UCL Institute of Education, United Kingdom \\ ${ }^{3}$ Serbian Society of Autism, Serbia \\ p.chevaliereutwente.nl
}

\begin{abstract}
We present design strategies for Human Robot Interaction for schoolaged autistic children with limited receptive language. Applying these strategies to the DE-ENIGMA project (large EU project addressing emotion recognition in autistic children) supported development of a new activity for in facial expression imitation whereby the robot imitates the child's face to encourage the child to notice facial expressions in a play-based game. A usability case study with 15 typically-developing children aged 4-6 at an English-language school in the Netherlands was performed to observe the feasibility of the setup and make design revisions before exposing the robot to autistic children.
\end{abstract}

Keywords: Robot, Child, Interaction, Prototype, Design, Mirroring, Imitation, Autism.

\section{Introduction}

Autism Spectrum Disorder (ASD) is characterized by difficulties in social interaction and communication and the presence of restricted and repetitive patterns of behaviours, interest or activities, as described in the DSM-5 [1]. Autistic children's affinity for robot, computer and mechanical components [2] has drawn the attention of social robotics researchers. Robots have been designed to enhance social engagement, imitation and joint attention skills in autistic children, with some encouraging results, including increased gaze toward the robot and increased number of smiles [3]-[5].

Population-specific needs and large individual differences [6] make designing robotic interactions for autistic children more challenging than designing for adult users or typically-developing children. For example, some autistic children show limited receptive language ability, so the specific words that the robot says are important. Several design strategies have been discussed in social robotics for autistic children. In [7], the authors describe the development process of tactile interaction with the robot KASPAR. They based their work on User Centred Principles which involves the target users of 
their work: autistic children and their therapist. In [8], the authors describe the development of a robot that plays various activities with an autistic child. The design was based on therapeutic and educational objectives identified by consulting experts.

In this paper, we described our approach to designing the dialogue for an innovative game for autistic children. This work is done within the DE-ENIGMA project (http://de-enigma.eu/), in which we aim to develop a novel intervention for teaching emotion recognition to autistic children through the use of Robokind's R25 model Zeno. This humanoid robot has an expressive humanlike face than enables it to produce different facial expressions. Emotion recognition is the overall goal for DE-ENIGMA because: (1) it is an important social skill and (2) autistic children have difficulties in recognising, understanding and producing facial expressions [9]. The DE-ENIGMA project involves experts in autism and in interactive robotics, and we describe here the different steps we followed to design a mirroring game as an entertaining way to prepare autistic children to notice and recognise different facial expressions.

In this paper, we propose a set of strategies for designing robot-based game content and dialogues for school-age autistic children. We then apply these strategies to the design of a new emotion-mirroring game with the Zeno robot, and present a qualitative usability study with 4-6 year old typically developing children. This is a first assessment of the validity of our strategies and mirroring game design.

The paper is structured as follows: Section 2 describes related work, section 3 presents our proposed guidelines to design new games, and section 4 presents the mirroring game we designed. The usability study is reported in Section 5. Section 6 concludes this paper.

\section{Related Work}

Prior strategies to help autistic children recognize and learn to respond appropriately to emotions include read-aloud social stories [10], films of animated transport vehicles inset with real-world video of emotional human faces (The Transporters series for young autistic children [11]), and social robots [12]-[14].

Researchers have developed different robotic platforms and different interaction strategies for teaching facial emotion expression recognition autistic children [12][15]. We observe three main interaction strategies in the aforementioned studies that use a robot to teach emotions. First, the robot displays a face and the child is asked which emotion she recognizes. Second, the robot displays a face and the child is asked to imitate the face. Third, a social story involving the robot is told to the child that reference facial expressions. These strategies result in activities being structured more as evaluations than as games: the robot first performs an action (a facial expression or a social story) and then the child has to give the correct answer, without providing space for exploration and play. Moreover, if unsuccessful, the child can lose interest or express anxiety [7].

Another strategy explored to teach emotions to autistic children is by facial imitation. Children seeing their face being imitated by another party can be an efficient strategy to combine game and social and emotion skill learning in autism. Previous work has 
reported that social responsiveness improved during and/or after a mirroring activity in which nonverbal autistic children were mirrored [9]. A potential reason for these benefit could be that explicit mirroring compensates for a dysfunctional mirror neuron system in autistic children [16].

Human Robot Interaction (HRI) studies have mainly explored imitation and mirroring through body gesture and found promising effects on children's social behaviors [3], [17]-[19]. In [3], autistic children who understood that the robot was mirroring their gestures manifested positive affect through vocalizations, suggesting a "robot mirror" may be entertaining. HRI studies exploring facial emotion expression imitation have mainly explored the child mirroring the robot rather than the robot mirroring the child. Finally, in [18], children were paired with either a robot or a human partner for an imitation task, involving body gestures and smiles. Autistic children paired with a robot spontaneously imitated smiles more than those paired with a human partner.

Modern computer vision and face tracking technology enables machine-interaction studies in which children see their face mirrored by a robot or virtual agent. The Emotion Mirror [20] is a game designed for autistic children based on mirroring and imitation of the face. In this game, the child can make facial expressions of basic emotions that are mirrored by a cartoon character on a screen or copy the character facial expression. In [21], the authors provide a technical description of their system to support recognition and production of facial expressions through a robotic platform and a small pilot study indicates positive development.

These studies suggest that mirroring activities might be appropriate and effective way for autistic children to learn about emotions. Here we propose adding a complementary step of having a robot mirror the facial expression of the child to support play. To support development of such an activity, we also present a set of interaction design strategies to fit the robot's speech to the needs of autistic children with limited receptive language ability.

\section{Developing autism-specific design strategies}

In this section, we describe the development of the mirroring game involving the expert teams in autism and in social robotics within the DE-ENIGMA project. We first conducted domain expert evaluation workshops with autism experts in order to identify possible game components and concepts, and identify requirements with respect to communicating with children and engaging them in interaction with robots. These workshops included (1) a series of initial paper prototypes, (2) reflection on child-robot interaction videos and educator interviews collected earlier in the project, (3) a review of existing interactive games for autistic children. Game concepts were further developed.

During the domain expert evaluation and the iterations on the game script, we defined a set of strategies for designing the dialogue of games for school age autistic children who have some receptive language. The following strategies were based on recommendations from the autism experts and our qualitative interviews/focus groups with educators. These strategies assume a child-robot interaction context where a supportive 
adult (i.e. teacher, parent, researcher) is continuously present, and available to support the interaction. While our current focus is emotion teaching, they may be applicable to child-robot interactions with other goals. The strategies are:

Defining the game objectives. A game can have many objectives in the child perspective (to learn a skill, to have fun, to relax...) or the researcher perspective (observe/trigger a certain behaviour).

Scaffolding. Scaffolding is an educational concept in which successively more difficult tasks and material are introduced, with a teacher providing temporary supports to help a learner "move up" to the next level of comprehension. For example, the child should first do easier versions of an activity before being offered adult or robot support to move to a more complex version. Also used in [15].

Demonstration. The adult should demonstrate each activity prior to the child engaging in the activity, to familiarise him/her with it, and facilitate learning.

Simple, short, non-ambiguous language. Word selection should be designed to match children's vocabulary. A simple phrase like "Let's make some faces" is preferable to a complex word like "mirroring." Instructions phrased as polite questions ("Can you move your mouth?") can be problematic because autistic children may answer them literally (i.e., saying "yes"). Requests for action, delivered with encouragement ("Move your mouth") are less ambiguous and easier to understand.

Pauses. Leave pauses between utterances and actions (adult or robot) so that children have time to process information.

Key phrases. Schools for autistic children often have key phrases that are used repeatedly across contexts (e.g. "Now you do it" to indicate the child's turn). We recommend teacher meetings or classroom observations to identify the key phrases used in a school or for a specific child. Repetition was also used in [18] for each set of instructions.

Positive feedback. Rewarding the child is very important as reinforcement of positive behaviour and/or good work, and for creating a positive interaction overall.

Cause and effect. To explicitly draw children's attention to the cause-and-effect relationship within the activity. In our project, we aim to teach the autistic children that emotions come from somewhere, and happen for a reason.

Predictability. The dialogue does not show variation over session, and is consistent within a session. For example, instructions for repeated trials should always be given using the exact same phrase (and ideally one that the child is known to understand).

\section{Proposed mirroring game}

\subsection{Game description}

We propose a face-mirroring game with the Zeno robot, incorporating the design strategies described in section 3. This game aims to: (1) familiarise an autistic child with Zeno in a gradual way; (2) prepare the child to pay attention to Zeno's facial features specifically; (3) prepare the child to generate facial expressions in response to Zeno (first any expression, then facial expressions that communicate appropriate expression); and (4) prepare the child to understand the cause-and-effect nature of mirroring (i.e., understand what "mirroring" actually is). 
The game setup is a triadic interaction between the child, an adult (i.e. a researcher or teacher) and the robot. The child sits in front of the robot, and the adult sits next to the child. The adult has a tablet that enables him/her to control the flow of the interaction with the robot via buttons. The adult's main goal is to scaffold the child's understanding of the cause-and-effect aspects of the activity, and narrate or label specific actions, expressions, and parts of the face. The mirror game steps are as follow:

1. Start: At the beginning of the session, the child is introduced to the therapist and invited to sit. The robot is covered or out of sight at this stage, so as not to be distracting.

2. Therapist models facial movements in mirror: To focus attention on the face and its features, the child and therapist play with an ordinary mirror. During this step, the therapist first models facial movements in the mirror, held so the child can see the reflection of the therapist's face moving. She/he narrates these actions to the child.

3. Child makes deliberate facial movements in mirror; therapist scaffolds causeand-effect understanding: Then, the therapist encourages the child to look at him/herself in the mirror and to deliberately move specific facial features, pointing out the cause and effect of the child's actions ("You move your mouth - Oh look! We can see your mouth moving!"). This first step enables us to have a baseline for the child's ability to deliberately move his/her face.

4. Child makes full-face expressions in mirror: Then, the therapist asks the child to make complete facial expression such as a "silly face" or a "monster face". The goal is for the child to create deliberate expressions of any kind and attend to these in the mirror, not to produce "correct" expressions.

5. Introduction to robot: The therapist introduces the child to the robot. The robot presents itself to the child, who is encouraged to greet the robot.

6. Robot mirrors child's face; therapist scaffolds cause-and-effect understanding: The therapist explains that the robot is going to "do the same face" as the child. As in step 3, the child is asked to move facial parts as the therapist describes the cause and effect ("You moved your mouth - Oh look! Zeno's mouth moves too! Zeno does the same"). If the child does not understand, the therapist can take the child's seat (to be in view of the cameras) and model the task for the child (as in step 2).

7. Child imitates robot facial expressions: Once a child understands step 6 , she/he is asked to mirror the robot's facial expression. The initial goal is for the child to make any deliberate imitation attempt, and then be gradually encouraged toward more specific, accurate imitation of the robot's face. The emotion labels of the robot's facial expressions are gradually introduced to the child during this step.

\subsection{Game dialogue}

A game dialogue was written following domain expert evaluation and the strategies above and revised with domain experts. The game dialogue (see Fig. 1 for an excerpt from the version used in testing) describes what the therapist and the robot say and do during a session, and takes into account the possible actions of the child. 
Fig. 1. Excerpt from dialogue for the mirroring game. Actions and dialogs are written in blue for the therapist and in green for Zeno, pause in red and additional notes on unexpected behaviour that can occurs in purple.

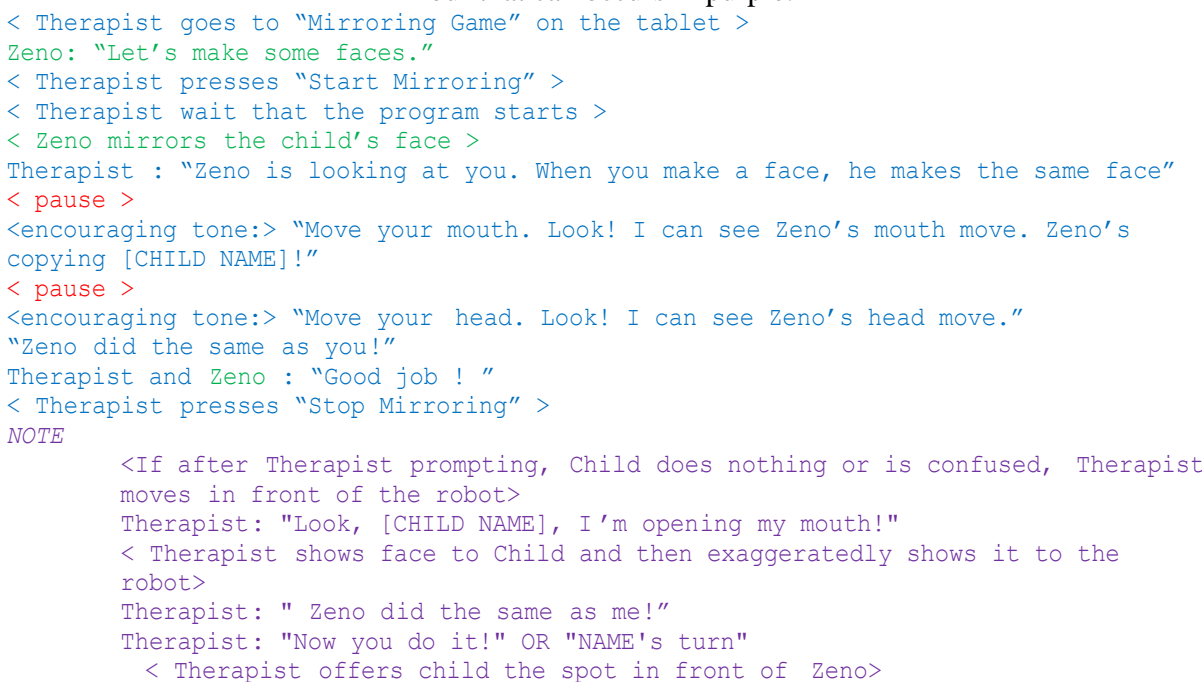

\subsection{Technical implementation}

The architecture of the child-robot interaction component of the mirroring game is composed of three modules: the Dialogue Manager (DM) (done with Flipper [22]), the Behaviour Realiser (BM) (done with ASAPRealizer [23]) and the Agent Control Engine (ACE) (done with the MechIO API of the Zeno robot and an android application for the tablet), as seen in Fig. 2. Please refer to [24] for details. The modules interacts with each other: (1) The DM generates behaviour specifications transmitted to the BR; (2) The BR converts the behaviour specifications into platform-specific commands to the ACE; (3) The ACE executes the commands and delivers feedback to the BR about planning and execution of the commands; (4) The BR provides higher level feedback to the DM about the behaviour progression.

Fig. 2 shows the connection between all our equipment. On the laptop, Flipper communicates the behavioural intent and receives feedback about the behaviour progression from AsapRealizer via a middleware (ActiveMQ Apollo). AsapRealizer sends the platform-specific commands to the tablet and robot and receives their feedback about the realisation of the behaviours The communication between the different hardware platforms goes via WiFi. AsapRealizer communicates with Zeno's controller through the MechIO API. The webcam is used for the mirroring game to perceive the child's face and is connected to the laptop via USB.

The face movements for mirroring activity were generated by a separate program design for the DE-ENIGMA project that used computer vision and facial landmark 
tracking [25] to identify and reproduce the relative positions of the child's eyes, eyebrows, lip corners, nose and mouth aperture.

Fig. 2. Diagram of the setup connections.

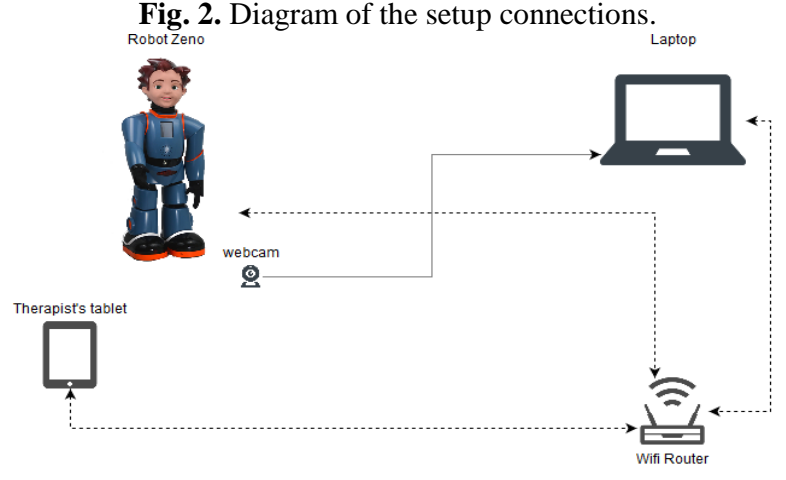

\section{$5 \quad$ Usability tests}

The mirroring game was first tested with typically-developing children, in order to gather initial information about its usability, robustness, and pacing. We are designing the game iteratively, and based on this first usability study the game will be revised, and evaluated with autistic children in the near future. The usability study was conducted at an international school in the Netherlands (i.e. school with English language instruction) with 15 typically-developing children between 4 and 6 years old (5 females).

Three children played the first mirroring game iteration with Zeno. Based on videos of their interactions, we modified the game to make the robot both more positive and more physically active (e.g. arm movement when it cheers the child's success, dancing at the end of the interaction).The "script" for the researcher's behaviour was also changed to improve the interaction.

The remaining 12 children played the second game iteration (now as described in Section 4.1). We report here our observations on the game steps. Step 2, in which the researcher shows his/her own face moving in the mirror to the child, was particularly helpful: some children were impressed by the experimental setting, and seeing the researcher doing funny expression could relax them and help them produce their own facial expressions. All children looked at the researcher's facial expression in the mirror. In steps 3 and 4 , in which the child was asked to make movements or facial expressions in the mirror, 10 out of the 12 children did try to make expressions when asked. In step 5, children met the robot, and all 12 children said hello when prompted.

In step 6, the robot mirrored children's faces. The mirroring algorithm was successful for 10 out of the 12 children, but was unable to successfully locate the faces of the remaining two. This may be due to hair covering most of the child's face, or because the child was standing too far from the camera. During the interaction, the mirroring was too sensitive for this context in the sense that the robot's servos tried to mirror every tiny change in the child's face. This resulted in the servos making constant noise, even when the servo didn't visibly move. 
Regarding children's understanding of mirroring, the 10 children correctly tracked all understood that the robot was mirroring their expressions in step 6. They also enjoyed it, with some children spontaneously laughing or remarking "Zeno is funny". One child additionally moved his arms to see if the robot was mirroring body gestures. The final activity step, in which the child attempts to mirror Zeno's face, was challenging for two of the youngest participants. They continued producing movements on their face for Zeno to mirror, or had difficulties focusing on Zeno's face. After that the researcher introduced this step ("Now, you do the same as Zeno"), the robot gave instructions with speech and mouth movements (i.e.: "Look at my face" - Zeno does a facial expression - "You do it!"). Some children imitated all Zeno's behaviour (including sounds), not only the facial expression of emotion. Overall, we observed that the game was feasible and enjoyable for typically-developing children of 4 to 6 years old during this short interaction. These positive reactions lead us to believe that autistic children, many of whom will be older than the text group, will also find this activity understandable, and able to scaffold their engagement with the robot's mirroring.

\section{Conclusions and Discussion}

In this paper, we propose specific strategies for HRI dialogues and interactions for school-aged autistic children. We recommend close collaboration between experts in autism and social robotics to develop activities that are understandable, and appropriately support this population. User sessions with teachers and with autistic children were performed in early phases of the DE-ENIGMA which led to initial prototypes of the overall interaction. Workshops with autism experts produced detailed strategies for dialogue design. A structured script was written and iterated that reflected lessons learned from teacher input, observation of autistic children and dialogue strategies developed from discussion with experts. An iterative usability study with typically-developing children has enabled us to observe the feasibility of the setup and make design revisions prior to evaluating these robot activities with autistic children. Our resulting design addresses the special needs of our user group and will be integrated in the robot. We anticipate the strategies mentioned above may be transferrable to other activities in which robots and autistic children are interacting, as these strategies were derived from a range of expert input and examples, and repurpose concepts originally used in educational and therapeutic interactions.

The next step in the DE-ENIGMA project is to conduct user studies of the mirroring activity with school-aged autistic children. We also have not explicitly compared robotbased mirroring to an adult mirroring the child,, since our primary goal was dialogue and interaction design rather than evaluating the effectiveness of the current activity as a teaching tool.

In future work, the DE-ENIGMA project aims to develop a more autonomous robot via signal processing and reasoning systems. We also aim to develop more strategies to account for children's different learning profiles. Finally, we want to address autistic children's sensory sensitivities, in the future development steps, by inhibiting noise and movements form the robot that could disturb the child. 


\section{Acknowledgments}

This publication has received funding from the European Union's Horizon 2020 research and innovation program under grant agreement No 688835 (DEENIGMA)

\section{References}

1. APA, Diagnostic and statistical manual of mental disorders (DSM-5®). American Psychiatric Pub, 2013.

2. M. Hart, "Autism/excel study," in Proceedings of the 7th international ACM SIGACCESS conference on Computers and accessibility, 2005, pp. 136-141.

3. A. Tapus et al., "Children with autism social engagement in interaction with Nao, an imitative robot-A series of single case experiments," Interaction studies, vol. 13, no. 3, pp. 315347, 2012.

4. K. Dautenhahn et al., "KASPAR-a minimally expressive humanoid robot for human-robot interaction research," Applied Bionics and Biomechanics, vol. 6, no. 3-4, pp. 369-397, 2009.

5. B. Vanderborght et al., "Using the social robot probo as a social story telling agent for children with ASD," Interaction Studies, vol. 13, no. 3, pp. 348-372, 2012.

6. E. Milne, "Increased intra-participant variability in children with autistic spectrum disorders: evidence from single-trial analysis of evoked EEG," Frontiers in psychology, vol. 2, p. 51, 2011.

7. B. Robins and K. Dautenhahn, "Developing Play Scenarios for Tactile Interaction with a Humanoid Robot: A Case Study Exploration with Children with Autism," in Social Robotics, 2010, pp. 243-252.

8. E. Ferrari, B. Robins, and K. Dautenhahn, "Therapeutic and educational objectives in robot assisted play for children with autism," in RO-MAN 2009-The 18th IEEE International Symposium on Robot and Human Interactive Communication, 2009, pp. 108-114.

9. A. Escalona, T. Field, J. Nadel, and B. Lundy, "Brief Report: Imitation Effects on Children with Autism," J Autism Dev Disord, vol. 32, no. 2, pp. 141-144, Apr. 2002.

10. "Wiley: Teaching Children with Autism to Mind-Read: A Practical Guide for Teachers and Parents - Patricia Howlin, Simon Baron-Cohen, Julie A. Hadwin." [Online]. Available: http://www.wiley.com/WileyCDA/WileyTitle/productCd-0471976237.html. [Accessed: 08-Jul-2017].

11. O. Golan et al., "Enhancing emotion recognition in children with autism spectrum conditions: An intervention using animated vehicles with real emotional faces," Journal of autism and developmental disorders, vol. 40, no. 3, pp. 269-279, 2010.

12. G. Pioggia et al., "Human-Robot Interaction in Autism: FACE, an Android-based Social Therapy," in RO-MAN 2007 - The 16th IEEE International Symposium on Robot and Human Interactive Communication, 2007, pp. 605-612.

13. C. A. Pop et al., "Can the social robot Probo help children with autism to identify situationbased emotions? A series of single case experiments," International Journal of Humanoid Robotics, vol. 10, no. 03, p. 1350025, 2013.

14. S. Costa, F. Soares, A. P. Pereira, C. Santos, and A. Hiolle, "A pilot study using imitation and storytelling scenarios as activities for labelling emotions by children with autism using a humanoid robot," in 4th International Conference on Development and Learning and on Epigenetic Robotics, 2014, pp. 299-304. 
15. G. Palestra, G. Varni, M. Chetouani, and F. Esposito, "A Multimodal and Multilevel System for Robotics Treatment of Autism in Children," in Proceedings of the International Workshop on Social Learning and Multimodal Interaction for Designing Artificial Agents, New York, NY, USA, 2016, p. 3:1-3:6.

16. A. F. de C. Hamilton, "Reflecting on the mirror neuron system in autism: A systematic review of current theories," Developmental Cognitive Neuroscience, vol. 3, pp. 91-105, Jan. 2013.

17. B. Robins, P. Dickerson, P. Stribling, and K. Dautenhahn, "Robot-mediated joint attention in children with autism: A case study in robot-human interaction," Interaction studies, vol. 5, no. 2, pp. 161-198, 2004.

18. A. Duquette, F. Michaud, and H. Mercier, "Exploring the use of a mobile robot as an imitation agent with children with low-functioning autism," Autonomous Robots, vol. 24, no. 2 , pp. 147-157, 2008.

19. P. Chevalier, G. Raiola, J.-C. Martin, B. Isableu, C. Bazile, and A. Tapus, "Do Sensory Preferences of Children with Autism Impact an Imitation Task with a Robot?," in Proceedings of the 2017 ACM/IEEE International Conference on Human-Robot Interaction, New York, NY, USA, 2017, pp. 177-186.

20. D. M. Deriso et al., "Exploring the Facial Expression Perception-Production Link Using Real-Time Automated Facial Expression Recognition," in Computer Vision - ECCV 2012. Workshops and Demonstrations, 2012, pp. 270-279.

21. V. Silva, F. Soares, and J. S. Esteves, "Mirroring and recognizing emotions through facial expressions for a RoboKind platform," in 2017 IEEE 5th Portuguese Meeting on Bioengineering (ENBENG), 2017, pp. 1-4.

22. M. ter Maat and D. Heylen, "Flipper: An Information State Component for Spoken Dialogue Systems," in Intelligent Virtual Agents, 2011, pp. 470-472.

23. D. Reidsma and H. van Welbergen, "AsapRealizer in practice - A modular and extensible architecture for a BML Realizer," Entertainment Computing, vol. 4, no. 3, pp. 157-169, Aug. 2013.

24. V. Vouloutsi et al., "Towards a Synthetic Tutor Assistant: The EASEL Project and its Architecture," in Biomimetic and Biohybrid Systems, 2016, pp. 353-364.

25. A. Asthana, S. Zafeiriou, G. Tzimiropoulos, S. Cheng, and M. Pantic, "From Pixels to Response Maps: Discriminative Image Filtering for Face Alignment in the Wild," IEEE Transactions on Pattern Analysis and Machine Intelligence, vol. 37, no. 6, pp. 1312-1320, Jun. 2015. 